\title{
Physical activity advice could become part of routine care for colorectal cancer survivors
}

\author{
"With significant collaborative efforts between research and clinical \\ teams, and with appropriate funding, physical activity could become \\ part of usual care for colorectal cancer patients."
}

\begin{abstract}
Abigail Fisher ${ }^{* \neq \neq, 1}$, Lee Smith ${ }^{\ddagger 1}$ \& Jane Wardle
\end{abstract}
First draft submitted: 21 September 2015; Accepted for publication: 29 September 2015; Published online: 30 November 2015

\begin{abstract}
Physical activity in colorectal cancer survivors

Colorectal cancer (CRC) is the fourth most common cancer in the UK [1]. In observational studies, regular participation in physical activity by CRC survivors has been found to be associated with positive health outcomes [2-4]. For example, in a recent meta-analysis of prospective cohort studies those who participated in high levels of physical activity after diagnosis (vs low levels) had a significantly lower risk (relative risk: 0.65; 95\% CI: 0.47-0.92) of CRC-specific mortality, and a similar inverse association was found for all-cause mortality [3]. However, despite potential benefits, physical activity levels in this population are low during and after treatment $[5,6]$.
\end{abstract}

Meta-analytic data from randomized controlled trials (RCTs) of physical activity in cancer survivors of mixed diagnoses (but mostly breast cancer) show improvements in many outcomes that are of particular importance to patients, including reductions in cancer-related fatigue and improved quality of life $[7,8]$. A recent review and meta-analysis of physical activity trials specifically in CRC found only five RCTs including a total of 238 patients [9], strongly highlighting the need for more research. However, despite the small numbers included, physical activity had strong positive effects on fitness [9]. An RCT to examine effects of physical activity on disease-related outcomes in CRC 'CHALLENGE' [10] is currently underway, and findings from this important trial have the potential to impact on guidelines for treatment. Simultaneously, 'real-world' pragmatic trials that demonstrate that physical activity can be embedded into usual care are required.

\section{Physical activity interventions in medical settings}

It is feasible that a physical activity recommendation from a health professional could influence behavior. Receiving physical activity advice in primary care has been shown to increase physical activity levels in sedentary adults [11]. Interestingly, emerging research suggests receiving advice from medical professionals on the benefits of physical activity may be an effective strategy to increase activity levels in cancer survivors. Fisher et al. investigated the association between recalling receiving physical activity advice and physical activity levels in a sample of 15,254 CRC

\section{KEYWORDS}

- advice $\bullet$ colorectal cancer

- medical professional • physical activity • survivor

'Department of Epidemiology \& Public Health, Health Behaviour Research Centre, University College London, London, WC1E 6BT, UK

*Author for correspondence: Tel.: +44 207679 1722; abigail.fisher@ucl.ac.uk

${ }^{\ddagger}$ Authors contributed equally

$\begin{array}{lll}\text { Future } & \text { fSS } \\ \text { Medicine } & \text { part of }\end{array}$ 
“...clinicians face a number of significant barriers to being able to give physical activity advice." survivors residing in England [12]. It was found that $31 \%$ of participants recalled receiving advice and those who recalled receiving this advice were more likely to participate in brisk physical activity $51 \%$ in the advice group vs $42 \%$ in the no advice group) and meet the physical activity guidelines ( 25 vs $20 \%$ ). An important point to note is that this study was cross-sectional and relied on patient recall. However, findings of an RCT in breast cancer survivors demonstrated that oncologist's advice significantly increased physical activity, although follow-up was only 5 weeks [13]. While a clinician's advice may be motivating in a patient enrolling in a physical activity program, for sustained behavior change to be observed, additional support would likely be required. One promising notion is the idea of using or adapting services that exist for other chronic conditions (e.g., exercise referral services). However, the efficacy of these services in increasing physical activity in CRC must first be demonstrated. Promising pilot work by Hubbard et al. in the UK suggests that cardiac rehabilitation exercise services may be feasible for use in CRC survivors [14], and this important work should be continued.

\section{When \& who should give advice on physical activity?}

Limited literature exists on when in the care pathway physical activity advice should be given to those diagnosed with CRC. CRC survivors express desire to increase physical activity levels [15], but the proportions who recall receiving any advice is currently low [12]. However, surveys are often limited by the use of retrospective recall, and valuable research could test the feasibility, acceptability and efficacy of physical activity advice and support at different points in the care pathway. Early intervention would be particularly important if research can demonstrate benefits of being fit before colorectal surgery, demonstrating that physical activity enhances responses to treatments or prevents disease progression (although these factors remain to be established in trials in patients with CRC and are an important area for future research). Additionally, patients with more advanced disease are an under-researched group in the lifestyle context, and trials examining the benefits in these patients would certainly be warranted.

Clinicians dealing with cancer survivors are in a good position to offer activity advice and they are often the patients' reported preferred source of information. For example, O'Leary et al. critically assessed the literature focusing on information use by early-stage breast cancer patients [16]. A total of 25 articles were included and the highest ranked information sources accessed and preferred were physicians. After receiving lifestyle advice from a medical professional a patient may be more motivated to change their behavior. In support, Russell et al.'s seminal study on the effect of general practitioners advice against smoking found that motivation and intention to stop smoking increased after receiving the advice [17]. However, clinicians face a number of significant barriers to being able to give physical activity advice. In a survey completed by 323 CRC clinicians in Scotland, only 52\% reported being familiar with any lifestyle advice for patients with CRC and many felt they lacked the skills, confidence and knowledge to discuss it [18]. One option may be to embed programs specifically designed to train health professionals in physical activity for cancer, such as the UK-based program CanRehab [19], in continued professional development.

Lack of specific guidelines are another barrier; in a survey of 274 oncology nurses working with CRC patients, being 'unsure what to recommend' was strongly related to whether they gave advice or not [20]. This is unsurprising, since the question of how much and what to recommend still remains unclear in CRC. However, until robust evidence exists that can define the doses required to improve health outcomes, it seems appropriate to follow the American College of Sports Medicine roundtable recommendations that cancer survivors should follow the guidelines of $\geq 150$ per week of at least moderate activity, and that some appropriate strength-based training is likely beneficial [21]. Any physical activity recommendation must take into account specific diagnosis, treatments, current condition, comorbidities and exercise history. However, it has been recommended that clinicians should at least advise all cancer survivors to 'avoid inactivity' where possible [21]. An additional (and not insignificant) barrier for clinicians in providing physical activity advice is lack of time, and this is a barrier that will likely increase, rather than diminish, in future.

Therefore, it is our role as researchers working in the field of physical activity oncology to bridge these significant gaps by conducting rigorous research to demonstrate beneficial effects of physical activity on disease-specific outcomes 
and identifying the appropriate dosages for CRC patients, and identifying/developing evidencebased referral pathways to enable clinicians to 'prescribe' physical activity for their CRC patients.

\section{Conclusion}

Evidence of the benefits of physical activity after a diagnosis of CRC are continually emerging. With significant collaborative efforts between research and clinical teams, and with appropriate funding, physical activity could become part of usual care for CRC patients.

\section{References}

1 Cancer Research UK. Bowel cancer incidence and statistics.

www.cancerresearchuk.org

2 Meyerhardt J, Heseltine D, Niedzwiecki D et al. Impact of physical activity on cancer recurrence and survival in patients with stage III colon cancer: findings from CALGB 89803. J. Clin. Oncol. 24, 3535-3541 (2006).

3 Je Y, Jeon J, Giovannucci E et al. Associations between physical activity and mortality in colorectal cancer: a meta-analysis of prospective cohort studies. Int. J. Cancer 133, 1905-1913 (2013).

4 Campbell P, Patel A, Newton C et al. Associations of recreational physical activity and leisure time spent sitting with colorectal cancer survival. J. Clin. Oncol. 31, 876-885 (2013).

5 Hawkes A, Lynch B, Owen N et al. Health behaviours of Australian colorectal cancer survivors compared with non-cancer population controls. J. Support. Care Cancer 16, 1097-1104 (2008).

6 Williams K, Steptoe A, Wardle J. Is a cancer diagnosis a trigger for health behaviour change? Findings from a prospective, population based study. Br. J. Cancer 108 , 2407-2412 (2013).

7 Speck RM, Courneya KS, Masse LC, Duval S, Schmitz KH. An update of controlled physical activity trials in cancer survivors: a

\section{Dedication}

In memory of Professor Jane Wardle (1950-2015).

\section{Financial \& competing interests disclosure}

$L$ Smith, J Wardle and A Fisher receive research support from The Cancer Research UK (grant number C1418/A141). The authors have no other relevant affiliations or financial involvement with any organization or entity with a financial interest in or financial conflict with the subject matter or materials discussed in the manuscript apart from those disclosed.

No writing assistance was utilized in the production of this manuscript. systematic review and meta-analysis. J. Cancer Surviv. 4, 87-100 (2010).

8 Fong DYT, Ho JWC, Hui BPH et al. Physical activity for cancer survivors: a meta-analysis of randomized controlled trials. BMJ 344, e70 (2012).

9 Cramer H, Lauche R, Klose R, Dobos G, Langhorst J. A systematic review and meta-analysis of exercise interventions for colorectal cancer patients. Eur. J. Cancer Care 23, 3-14 (2014).

10 Courneya KS, Booth MD, Gill S et al. The Colon Health and Life-Long Exercise Change trial: a randomized trial of the National Cancer Institute of Canada Clinical Trials Group. Curr. Oncol. 15, 262-270 (2008).

11 Calfas K, Long B, Sallis J et al. A controlled trial of physician counselling to promote the adoption of physical activity. Prev. Med. 25, 225-233 (1996).

12 Fisher A, Williams K, Beeken R et al. Recall of physical activity advice was associated with higher levels of physical activity in colorectal cancer patients. BMJ Open 5, e006853 (2015).

13 Jones LW, Courneya KS, Fairey AS et al. Effects of an oncologist's recommendation to exercise on self-reported behaviour in newly diagnosed breast cancer survivors: a single-blind randomized trial. Ann. Behav. Med. 29, 105-113 (2004).
14 Munro J, Adams R, Campbell A et al. CRIB-the use of cardiac rehabilitation services to aid the recovery of patients with bowel cancer: a pilot randomised controlled trial (RCT) with embedded feasibility study. BMJ Open 4, e004684 (2014).

15 Dennis DL, Waring JL, Payeur N, Cosby C, Daudt HML. Making lifestyle changes after colorectal cancer: insights for programme development. Curr. Oncol. 20, e493-511 (2013).

16 O’Leary K, Estabrooks C, Olson K et al. Information acquisition for woman facing surgical treatment for breast cancer: influencing factors and selected outcomes. Patient Educ. Couns. 69, 5-19 (2007).

17 Russell M, Wilson C, Taylor C et al. Effect of general practitioners' advice against smoking. Br. Med. J. 2(6184), 231-235 (1979).

18 Anderson AS, Caswell S, Wells M, Steele RJ. Obesity and lifestyle advice in colorectal cancer survivors - how well are clinicians prepared? Colorectal Dis. 15, 949-957 (2013).

19 CanRehab. www.canrehab.co.uk

20 Karvinen KH, McGourty S, Parent T et al. Physical activity promotion among oncology nurses. Cancer Nurse 35, e41-8 (2012).

21 Schmitz KH, Courneya KS, Matthews C et al. American College of Sports Medicine round table on exercise guidelines for cancer survivors. Med. Sci. Sports Excer. 42, 1409-1426 (2010). 